\title{
Extending the limits of transcatheter closure of atrial septal defects with the double umbrella device (CardioSEAL)
}

\author{
R Kaulitz, T Paul, G Hausdorf
}

\begin{abstract}
Objective-To report initial findings from a selected group of patients with morphological variations of the atrial septal defect who underwent transcatheter closure with a second generation redesigned double umbrella device.
\end{abstract}

Patients-Two patients with abnormal location of the oval fossa and partial deficiency of the septal rim, three patients with multiple defects, and two patients with a multiperforated aneurysm of the interatrial septum (age range, 3.6-25.5 years).

Methods-Defects were closed with the double umbrella device (CardioSEAL) consisting of two sets of flexible arms (with central and two mid-arm hinges) covered with sewn Dacron patches. The implantation procedure was monitored by transoesophageal echocardiography.

Results-The diameter of the defect measured during transoesophageal echocardiography ranged from 7-18 mm and the balloon stretched diameter ranged from 13-21 mm. The size of the devices varied from $28-33 \mathrm{~mm}$ and the ratio of device size to defect size varied from 1.6-2.1. Two devices ( 23 and $28 \mathrm{~mm}$ ) were chosen in a patient with two separated defects. No complications or serious arrhythmias were observed during implantation or follow up (median, 1.8 months). Residual shunting was trivial in three patients and mild in one patient (inferiorly located additional defect).

Conclusions-To extend the selection critera of an isolated central interatrial defect for transcatheter closure, some modifications of the implantation technique are needed. Using the redesigned double umbrella device, effective closure in patients with multiple or irregularly shaped atrial septal defects was achieved, indicating a broadening of the spectrum of transcatheter closure.

(Heart 1998;80:54-59)

Keywords: atrial septal defects; transcatheter closure; congenital heart disorders; double umbrella device; CardioSEAL

Percutaneous non-surgical closure is an alternative to operative repair of a serious atrial septal defect or patent foramen ovale associated with presumptive paradoxic embolism. Major limitations for transcatheter closure can result from the size and location of the defect, insufficient extension of septal margins, and associated partial anomalous pulmonary venous drainage. Several devices have been designed for transcatheter occlusion and feasibility, effectiveness, and safety have been demonstrated. ${ }^{1-5}$ The CardioSEAL septal occluder was developed as a second generation improved and refined redesign of the double umbrella device (Clamshell occluder), ${ }^{6}$ which had been associated with a high rate of device arm fractures during long term follow up. ${ }^{7}$

We report our initial experience in a selected group of patients who presented with atrial septal defects other than deficiencies within the oval fossa. Patients had either an abnormal superior or inferior location of the oval defect and partial deficiency of the septal rim, multiple atrial septal defects, or multiperforated aneurysms of the interatrial septum. These morphological variations precluded the patients from catheter closure by conventional occluder systems.

\section{Methods}

STUDY PROTOCOL

Patients or guardians agreed to participate in a limited clinical trial; guidelines for the study were approved by the authorised human subjects committee and by the local governmental authorities. Only patients who needed surgical correction were included.

PATIENTS

Between October 1996 and June 1997, 28 patients (age range, 3.4-25.5 years; median, 6.3) underwent transcatheter atrial septal defect closure with the double umbrella device (CardioSEAL; Nitinol Med Techn Inc, Boston Massachusetts, USA). Seven of these patients presented with morphological variations, abnormal location of the oval fossa, multiple defects, or a multiperforated atrial septal aneurysm and are the subjects of our study. Patients with a sinus venosus-type defect, ostium primum, or coronary sinus defect and associated partial anomalous venous drainage were excluded. Data on patients undergoing transcatheter occlusion are provided in table 1 .

Potential preprocedural and postprocedural atrial arrhythmias and conduction delay were evaluated by standard 12 lead electrocardiograms (ECGs) and Holter recordings to evaluate the non-invasive electrophysiological findings after placement of a double umbrella device. ${ }^{8} \mathrm{~A}$ standard right heart catheterisation was performed for haemodynamic evaluation 
and to exclude other associated cardiac lesions, especially partial anomalous pulmonary venous drainage. Pressure measurements were performed for estimation of haemodynamic significance and oximetry for assessment of pulmonary to systemic flow ratio.

DEVICE DESIGN AND IMPLANTATION TECHNIQUE The device consists of two interconnected umbrellas (umbrella size (arm length) $\leqslant 40 \mathrm{~mm}$ ). Both sets of flexible, spring loaded arms (four arm MP35N wire frame with central and two mid-arm hinges) are covered with sewn Dacron patches.

Principles of the methods for implantation of a double umbrella device have been described previously. ${ }^{6}$ For selection of the appropriate occluder device, all patients underwent catheter sizing to establish the stretched diameter defined by measuring the maximum balloon diameter occluding the defect. ${ }^{9} 10$ An implant to stretched diameter ratio of $1.7-2.0$ to 1 was recommended for implantation. The collapsed double umbrella device and the $11 \mathrm{~F}$ sheath as a unit are positioned on the left atrial side of the defect. By partially pulling back the sheath the left atrial arms are opened. The sheath, delivery system, and left atrial umbrella are retracted to bring at least one arm into slight contact with the septum. The sheath is pulled back further to allow it to open the right atrial umbrella. After confirmation of appropriate arm and occluder position the device is released, straddling the atrial septum.

The implantation procedure with subsequent delivery of the distal and proximal umbrella, adequate positioning of the device, and release from the guidewire was monitored by transoesophageal echocardiography. Postplacement studies were performed to rule out systemic or pulmonary venous obstruction or compromise of the atrioventricular valve function and to assess the presence of potential residual shunting.

TWO DIMENSIONAL ECHOCARDIOGRAPHY

For preinterventional screening, patients were investigated with transthoracic echocardiography (Hewlett-Packard Sonos 1000 ultrasound system using a 3.5 to $5.0 \mathrm{MHz}$ phased array transducer). All possible views from the apical, parasternal, and subcostal region were obtained to define the position and the dimension of the atrial septal defect and to identify multiperforated atrial septal defects. In addition, the inferior and superior septal tissue rims and the length of the interatrial septum were measured. ${ }^{11}$

Transoesophageal echocardiography (5.0 $\mathrm{MHz}$ biplane probe) just before cardiac catheterisation was used to confirm and complete the transthoracic examination and measurements. All patients were investigated under general anaesthesia to prevent vigorous patient respiration and movement and to allow precise device placement. ${ }^{12}$ Transoesophageal echocardiography was used for continuous monitoring and guidance of the procedure. Four chamber views and long axis views were obtained by moving the transoesophageal probe slowly in the cranio-caudal direction. Evaluation of septal geometry and size as well as measurements of the defect size in the transversal and longitudinal plane are required. Positioning of the long sheath and delivery system was monitored. Opening of the left atrial arms and their approximation to the interatrial septum were continuously visualised on transoesophageal echocardiography. Exclusion of left atrial arm prolapse was mandatory before opening of the right atrial arms. The appropriate arm and occluder position was documented and, after release of the system, residual leakages were investigated by colour flow mapping.

Residual shunting detected by colour flow mapping studies was graded as trivial (minimal jet of colour disturbance on the right atrial side), mild (width of colour Doppler jet 1-2 $\mathrm{mm}$ extending into the right atrium), and moderate (large Doppler jet on colour flow mapping, defect visible on cross sectional echocardiography, signs of right ventricular volume overload).

FOLLOW UP

Before discharge, clinical evaluation, chest roentgenogram, ECG and Holter monitoring, and transthoracic echocardiography were performed. Regular follow up after 1, 6, and 12

Table 1 Data on patients undergoing transcatheter occlusion of atrial septal defect, echocardiographic measurements, and implantation data

\begin{tabular}{|c|c|c|c|c|c|c|c|c|c|c|c|}
\hline \multirow[b]{2}{*}{ Patient } & \multirow[b]{2}{*}{$\begin{array}{l}\text { Age } \\
\text { (years) }\end{array}$} & \multirow[b]{2}{*}{$\begin{array}{l}\text { Weight } \\
(\mathrm{kg})\end{array}$} & \multirow[b]{2}{*}{ Anatomy } & \multicolumn{3}{|c|}{ Size of $A S D^{\star}$} & \multirow{2}{*}{$\begin{array}{l}\text { Septal } \\
\text { length } \\
(\mathrm{mm})\end{array}$} & \multirow{2}{*}{$\begin{array}{l}\text { Implanted } \\
\text { device } \\
(\mathrm{mm})\end{array}$} & \multicolumn{2}{|c|}{ Ratio device size } & \multirow[b]{2}{*}{$\begin{array}{l}\text { Residual } \\
\text { shunt }\end{array}$} \\
\hline & & & & $\begin{array}{l}T T E \\
(m m)\end{array}$ & $\begin{array}{l}T O E \\
(m m)\end{array}$ & $\begin{array}{l}\text { Balloon } \\
(\mathrm{mm})\end{array}$ & & & Balloon & IAS & \\
\hline 1 & 3.6 & 11.2 & Multiperforated aneurysm of the oval fossa & 13 & 12 & 17 & 30 & 33 & 1.9 & 1.1 & Trivial \\
\hline 2 & 4.2 & 17 & $\begin{array}{l}\text { Multiperforated aneurysm with an eccentric large } \\
\text { defect }\end{array}$ & 9 & 12 & 13 & 32 & 28 & 2.1 & 0.87 & - \\
\hline 3 & 25.5 & 50 & $\begin{array}{l}\text { Multifenestrated atrial septal defect (central } \\
\text { secundum defect with fenestrated septum } \\
\text { primum covering the oval fossa) }\end{array}$ & 6 & 7 & 17 & 38 & 28 & 2.0 & 0.73 & - \\
\hline 4 & 4.6 & 18 & $\begin{array}{l}\text { Multifenestrated atrial septal defect (central } \\
\text { secundum defect with fenestrated septum } \\
\text { primum covering the oval fossa) }\end{array}$ & 9 & 10 & 16 & 33 & 33 & 2.1 & 1.0 & Trivial \\
\hline 5 & 5.0 & 21.7 & $\begin{array}{l}\text { ASD adjacent to the entrance of the SCV, lack of } \\
\text { anterior rim }\end{array}$ & 6 & 10 & 13 & 37 & 28 & 2.1 & 0.75 & - \\
\hline 6 & 7.3 & 24.5 & Defect size $>20 \mathrm{~mm}$, lack of superior rim & 10 & 18 & 21 & 35 & 33 & 1.6 & 0.94 & Trivial \\
\hline 7 & 13.3 & 56 & $\begin{array}{l}\text { ASD inferiorly located in front of the mouth of the } \\
\text { ICV, additional atrial septal defects }\end{array}$ & 10 & 10 & 14 & 44 & $23 / 28$ & - & - & $\begin{array}{l}\text { Mild- } \\
\text { moderate }\end{array}$ \\
\hline
\end{tabular}

${ }^{\star}$ Largest diameter

ASD, atrial septal defect; ICV, inferior caval vein; SCV, superior caval vein; TTE, transthoracic echocardiography; TOE, transoesophageal echocardiography; balloon, balloon stretched diameter; IAS, interatrial septum length. 
months after closure is necessary. Aspirin $(2-3 \mathrm{mg} / \mathrm{kg}$ daily) is recommended for six months after occlusion.

\section{Results}

During a period of eight months, 28 patients were scheduled for interventional closure of an atrial septal defect. There were 21 patients with an isolated secundum atrial septal defect. The seven patients in this study presented with a particular morphological variation of the septal defect with regard to extension and location or multiple atrial septal defects. Data on preinterventional transthoracic and transoesophageal echocardiography and implantation are provided in table 1 .

MODIFICATION OF DEVICE IMPLANTATION

To extend the selection critera of an isolated central interatrial septum defect (secundum atrial septal defect) for transcatheter closure some modifications of the implantation technique are needed. In patient 1 , who had multiple perforations, it was necessary to pass the centre of the aneurysm to place the sheath. This enabled safe positioning of the occluder system and guaranteed occlusion of nearly all of the multiple defects by the left atrial umbrella. In patient 2 , the eccentrically located largest defect within the fenestrated aneurysm was passed with the delivery system. Complete occlusion was achieved (fig 1). In patients 3 and 4 , small atrial septal defects in addition to a central secundum-type atrial septal defect required repeated rotation of the device after opening of the left atrial arms to achieve optimal contact with the septum and to cover the additional defect. To position the occluder system safely in patient 5 , who had a superiorly located atrial septal defect and a lack of the superior and anterior rim, one arm of the right atrial umbrella was fixed in the mouth of the superior caval vein by rotating the whole system before release (fig 2). Initial minimal residual shunt at the rim of the device immediately after implantation was not observed during follow up. Patient 6 , who had a large defect of the oval fossa, also had inadequacy of a muscular rim around the defect and lacked the anterior rim. The left atrial umbrella was positioned with the Dacron patch between two arms covering the area of incomplete rim. In the same region, trivial residual shunting was detected on follow up colour Doppler studies. Patient 7 was found to have an inferiorly located large atrial septal defect and at least two small defects. As one centrally positioned device could not simultaneously cover the inferior and superior part of the septum, we decided to place two devices. First, a small superiorly located defect was occluded by positioning the distal part of the device so that the more medially located defect was also occluded by the left atrial umbrella. After release of the first device, the inferiorly located defect was sized and an appropriate device was chosen. Both devices showed little overlapping of their inferior and superior arms, respectively (fig 3). Mild residual shunting at the inferior rim of the caudally positioned device was observed immediately after the procedure and during follow up.

\section{OUTCOME}

No complications were observed during implantation. During follow up (range, 0.24-3.8 months; median 1.8), none of the patients had device embolisation, thromboembolic events, fractures of the implanted device, pericardial effusion, signs of systemic or pulmonary venous obstruction, or impaired atrioventricular valve function. Non-sustained supraventricular arrhythmias were observed during the procedure in one patient (patient 3). In every patient, Holter monitoring was performed preintervention and postintervention. Apart from preinterventionally documented isolated supraventricular ectopy, no arrhythmias were detected. All patients were in sinus rhythm and no second or third degree atrioventricular block occurred. Apart from one patient who had mild bleeding from the puncture site, no bleeding complications occurred during continuous heparinisation for the first 24 hours and during the early postinterventional period.
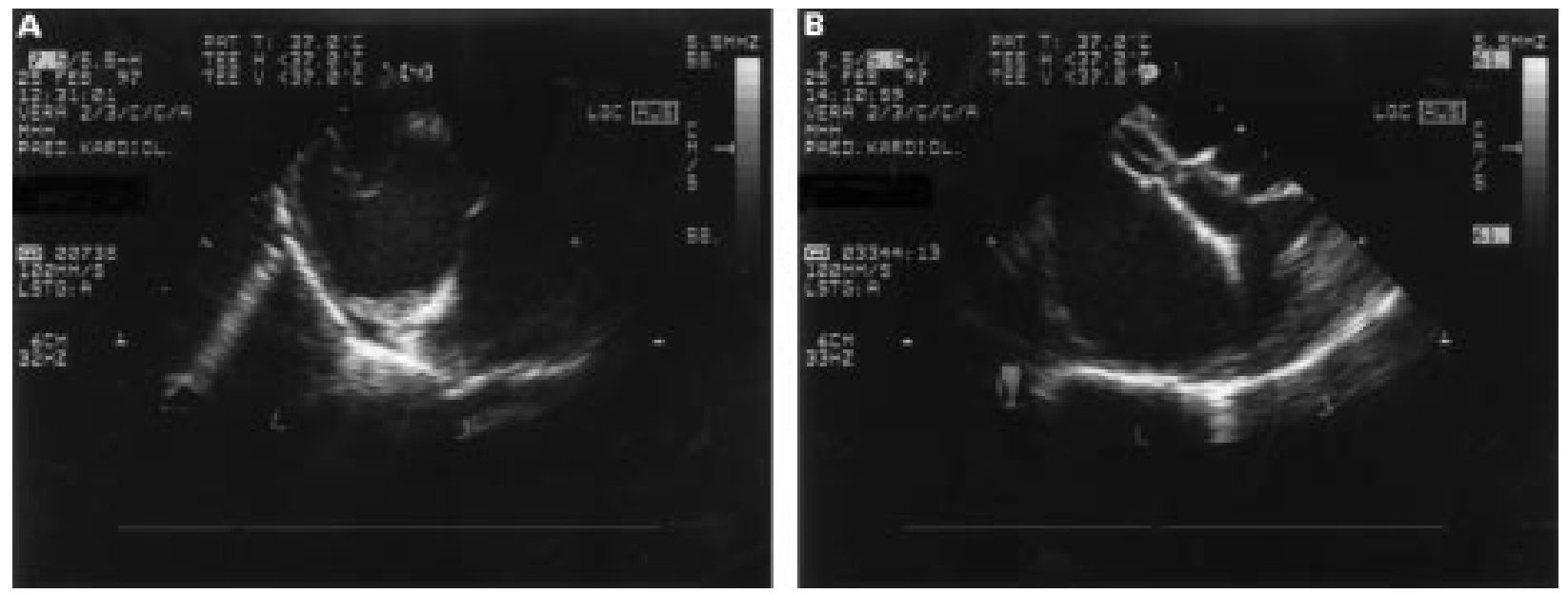

Figure 1 Transoesophageal echocardiography (A) before and (B) after occlusion in a patient with a multiperforated aneurysm. 

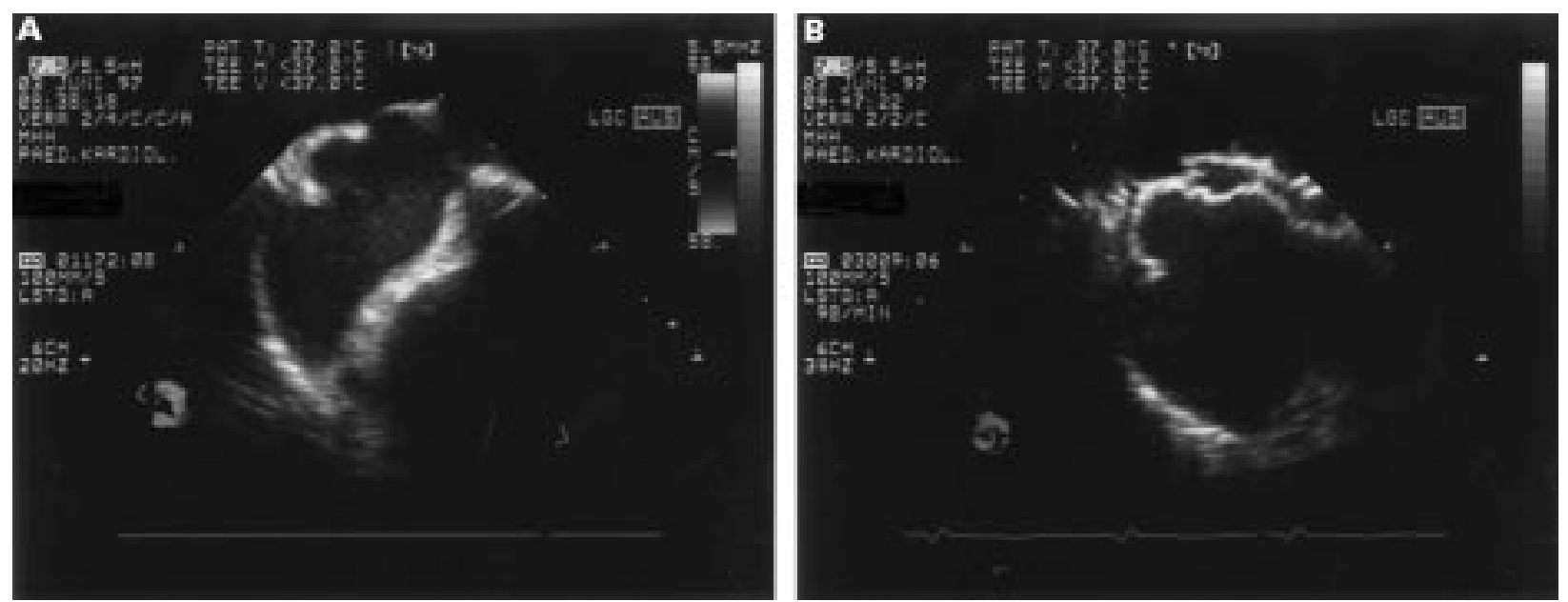

Figure 2 Selected video frame from transoesophageal echocardiographic study of patient 6 (transverse plane), in whom (A) lack of superior and anterior defect rim required $(B)$ safe positioning of one right atrial arm of the device in the mouth of the superior caval vein before releasing the device.
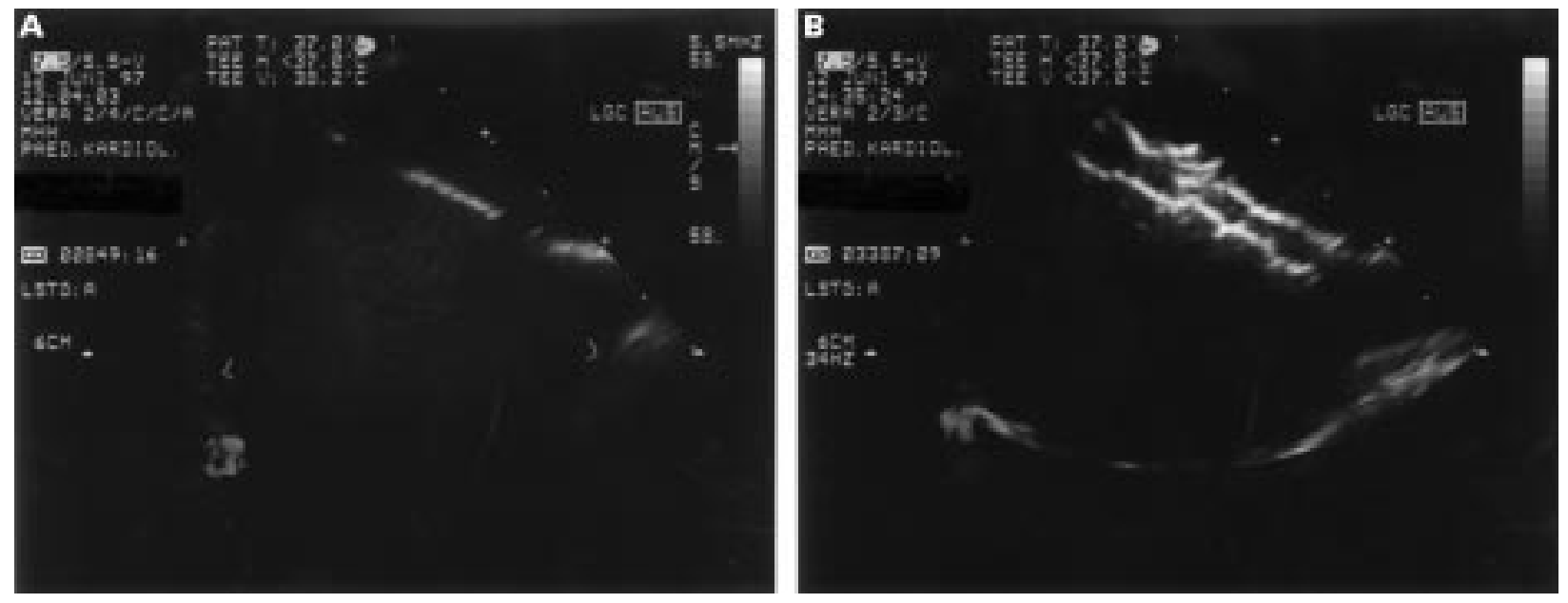

Figure 3 (A) Transoesophageal echocardiographic imaging of patient 7 (longitudinal plane) of the large inferiorly located septal defect and additionally smaller defect. (B) Imaging immediately after release of the second inferiorly located device. There was little overlapping of the distal arms of both devices.

\section{Discussion}

Transcatheter closure of atrial septal defects has become an alternative to surgery in selected patients. Several devices are available but long term experience is still lacking. ${ }^{16}{ }^{13} 14$ Interventional closure remains impossible in patients with ostium primum defects, sinus venosus defects, and secundum defects $>25 \mathrm{~mm}$. There are relative contraindications in patients with: multiple atrial septal defects; lack of a rim around the defect, which increases the risk of device embolisation; short septal length; small left atrium; and unusual location of the defect. ${ }^{1516}$ These morphological patterns of the atrial septal defect were thought to interfere with closure by currently available devices and to preclude transcatheter closure. Using the recently redesigned double umbrella device we achieved effective closure in a group of patients with multiple or irregularly shaped and located atrial septal defects; thus, broadening the spectrum of transcatheter closure.

\section{PATIENT SELECTION}

Preinterventional transthoracic echocardiography is performed routinely in our patients to confirm suitability and to detect relative contraindications for transcatheter closure, because these might change the catheterisation technique. Reproducible measurements of interatrial septal length determine the maximum diameter of the device that can be implanted without the risk of damage to adjacent cardiac structures. Abnormal location of the atrial septal defect usually becomes obvious during transthoracic echocardiography using multiple transducer positions. However, depending on the age of the patient and potentially restricted echo windows, it can be difficult to differentiate a large atrial septal defect (suggesting the need for surgical correction) from a multiperforated interatrial septum. In this situation, as well as in patients presenting with insufficient development of the muscular rim around the defect, preinterventional detailed transoesophageal echocardiography is mandatory to confirm the suitability of patients for transcatheter closure. Lack of superior, inferior, or anterior rim around the defect might increase the risk of embolisation and residual shunting. ${ }^{6}$ Thus, objective criteria that could predict successful occlusion of the atrial septal defect are needed. However, in a study with the buttoned device, when several echocardiographic parameters were investigated, only defect size $<15 \mathrm{~mm}$, a ratio of defect size to septal length less than 
0.35 , and a ratio of superior rim to defect size larger than 0.75 were predictive of successful closure. ${ }^{11}$ With regard to these parameters, size of the largest defect, as measured on transthoracic echocardiography, was $<15 \mathrm{~mm}$ in all of our patients; however, the ratio to septal length was $<0.35$ in none of the patients. In another clinical study with the double umbrella device, only an atrial septal defect size $<13 \mathrm{~mm}$, as measured on transoesophageal echocardiography, was predictive for effective closure. ${ }^{17}$ However, no information was given on the balloon stretched diameter or atrial septal length to define the appropriate, and estimate the largest, device that could be implanted.

\section{BALLOON SIZING}

Sizing of the atrial septal defect can be done by angiography, echocardiography, and balloon sizing. The final decision to implant the device and the selection of device size is now based largely on balloon sizing of the atrial septal defect and the test for complete occlusion of the defect during cardiac catheterisation. ${ }^{9}{ }^{18}$ The risk of distension and overestimation of the defect size and dependance on texture and thickness of the interatrial septum are potential sources of error in determining defect size.

Larger devices than calculated from balloon sizing were chosen in three of our patients with multiple atrial septal defects (patients 2 and 4) and an abnormally superior located defect (patient 5). This guaranteed the occlusion of the additional defects and safe positioning of the device, respectively. None of the potential complications associated with balloon sizing (arrhythmias, obstruction of mitral inflow or venous return, distension of the septal defect $)^{10}$ occurred, even when a relatively large balloon was used.

\section{DEVICE IMPLANTATION}

The technique of implantation of a double umbrella device used principally in patients with an isolated central atrial septal defect needed some modifications with regard to the unusual types of defects in our patients. ${ }^{6}{ }^{15}$ In most patients, repositioning of the device was necessary under echocardiographic guidance. This allowed optimal positioning of the left atrial umbrella, safe exclusion of left atrial arm prolapse, and, ideally, positioning with only one arm in slight contact with the interatrial septum before right atrial arm deployment. As the CardioSEAL device is not self centring, positioning of the device as demanded by the shape and location of the defect was possible. This was particularly important and advantageous in both patients with a multiperforated aneurysm of the interatrial septum to achieve occlusion of additional defects. In one patient with an abnormally superior located defect and lack of anterior rim, rotation of the device and positioning of one right atrial arm in the mouth of the superior caval vein allowed safe device deployment. This indicates that an adequate rim of septal tissue surrounding the whole defect is no longer mandatory.

For our patients, the mean (SD) ratio of the implanted device to atrial septal length was 0.9
(0.13) (range, 0.74-1.1), indicating the need for relatively large devices because of inclusion of patients with multiple atrial septal defects. In one patient, size and location of two dominant defects required the implantation of two devices, which covered almost the total septal length (patient 7). Inferiorly located mild residual shunting was detected on postinterventional echocardiography. This patient was the first in whom safe and effective deployment of two devices has been performed. Interference with adjacent structures was not seen in this patient or in the other three patients in whom a device of nearly the same size as the interatrial septal length was implanted.

RESIDUAL SHUNTING

During follow up, identification of presence or absence of residual shunting in patients undergoing transcatheter closure might be difficult using transthoracic echocardiography alone. Late residual shunting might occur because of initial incomplete endothelialisation or progressive instability of the device. A regular transoesophageal echocardiographic follow up is recommended during long term follow up.

\section{POTENTIAL COMPLICATIONS}

Procedural complications such as device embolisation, air embolism, alteration of atrioventricular valve function, or obstruction of venous return were not observed in this initial series. The risk of late metal fatigue fractures as described for the clamshell occluder is thought to be much lower for this redesigned double umbrella device, as described recently in vitro. ${ }^{7}$ Important factors are the MP35N wire frame and the device configuration, with two mid-arm hinges on each arm. These factors were the main reasons for broadening the spectrum of atrial septal closure in our study.

This initial experience with transcatheter closure using the double umbrella device in seven patients who presented with morphological variations of the atrial septum considered previously to be a relative contraindication for device closure, was encouraging. However, long term follow up and further evaluation in these special cases is needed.

1 Rao PS, Sideris EB, Hausdorf G, et al. International experience with secundum atrial septal defect occlusion by
the buttoned device. Am Heart $\mathcal{F}$ 1994;128:1022-35.

2 Redington AN, Rigby ML. Transcatheter closure of interatrial communications with a modified umbrella device. $\mathrm{Br}$ Heart f 1994;72:372-7.

3 Ende DJ, Chopra PS, Rao PS. Transcatheter closure of Ende DJ, Chopra PS, Rao PS. Transcatheter closure of
atrial septal defect or patent foramen ovale with the atrial septal defect or patent foramen ovale with the
buttoned device for prevention of recurrence of paradoxic embolism. Am f Cardiol 1996; 78:233-6.

4 Bridges ND, Hellenbrand W, Latson L, et al. Transcatheter closure of patent foramen ovale after presumed paradoxical embolism. Circulation 1992;86:1902-8.

5 Justo RN, Nykanen DG, Boutin C, et al. Clinical impact of transcatheter closure of secundum atrial septal defects with the double umbrella device. Am f Cardiol 1996;77:889-92.

6 Rome JJ, Keane JF, Perry SB, et al. Double-umbrella closure of atrial defects: initial clinical application. Circulation 1990;82:1044-5.

7 Koike K, Echigo S, Kumate M, et al. Transcatheter closure of atrial septal defect with a prototype clamshell septal umbrella: one year follow-up. $\mathcal{F}$ Cardiol 1994;24:53-60.

8 Schenck MH, Sterba R, Foreman CK, et al. Improvement in noninvasive electrophysiologic findings in children after noninvasive electrophysiologic findings in children after 1995;76:695-8.

9 King TD, Thompson SL, Mills NL. Measurement of atrial septal defect during cardiac catheterization. Experimental and clinical results. Am f Cardiol 1978;41:537-42. 
10 Rao PS, Langhough R. Relationship of echocardiographic, shunt flow and angiographic size of the stretched diameter of atrial septal defect. Am Heart f 1991;122:505-8.

11 Reddy SC, Rao PS, Ewenko J, et al. Echocardiogrphic predictors of success of closure of atrial septal defect with the buttoned device. Am Heart f 1995;129:76-82.

12 Hickey PR, Wessel DL, Streitz SL, et al. Transcatheter closure of atrial septal defects: hemodynamic complications and anesthetic management. Anesth Analg 1992;74:44-50

13 Das GS, Voss G, Jarvis G, et al. Experimental atrial septal defect closure with a new self-centering device. Circulation 1993;88:1754-64

14 Hausdorf G, Schneider M, Franzbach B, et al. Transcatheter closure of secundum atrial septal defects with the atrial septal defect occlusion system (ASDOS): initial experience in children. Heart 1996;75:83-8.
15 Boutin C, Musewe NM, Smallhorn JF, et al. Echocardiographic follow-up of atrial septal defect after catheter closure by double-umbrella device. Circulation 1993;88: 621-7.

16 Ferreira SM, Ho SY, Anderson RH. Morphological study of defects of the atrial septum within the oval fossa: implications for transcatheter closure of left-to-right shunt. Br Heart f 1992;67:316-20.

17 Rosenfeld HM, van der Velde ME, Sanders SP, et al. Echocardiographic predictors of candidacy for successful transcatheter atrial septal defect closure. Cathet Cardiovasc Diagn 1995;34:29-34.

18 Godart F, Rey C, Francart C, et al. Two-dimensional echocardiographic and color Doppler measurements of atrial septal defect, and comparison with the balloonstretched diameter. Am f Cardiol 1993;72:1095-7.

\section{IMAGES IN CARDIOLOGY}

\section{Right sided intracardiac thrombus}

This echocardiogram was recorded from a 62 year old woman who presented with breathlessness and pleuritic chest pain. Three weeks before admission she had sprained her ankle and been forced to rest. Subsequently she noticed calf swelling, followed by a presyncopal episode just before the onset of her presenting symptoms. On admission, examination of her leg and Doppler studies of leg veins were normal. However, she had signs of right heart fail-

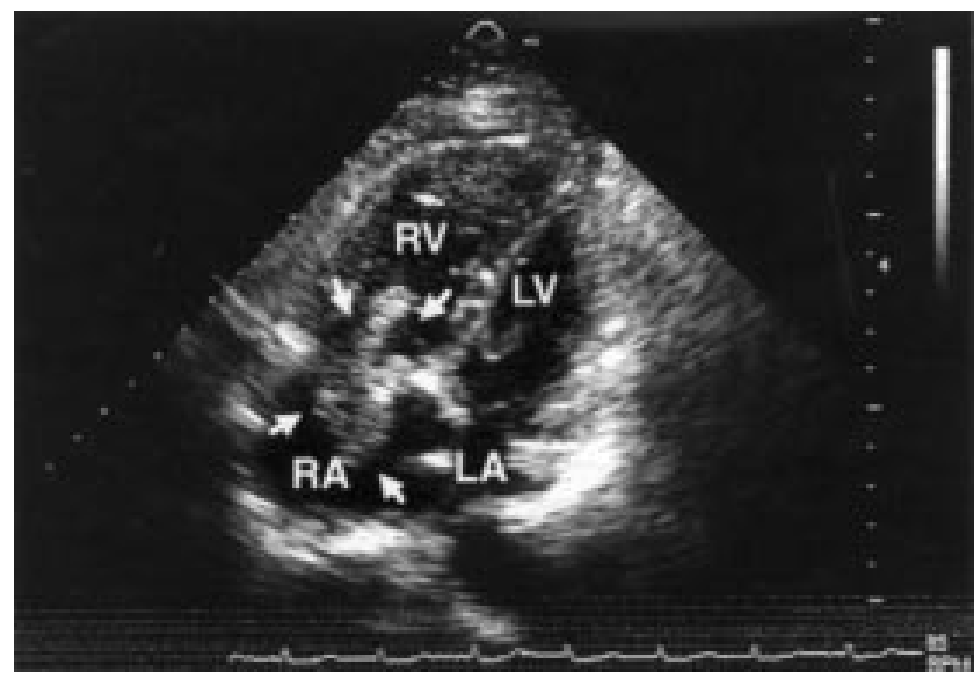

ure and an electrocardiographic pattern of right heart strain. Chest radiography was normal. On the basis of the echocardiogram a diagnosis of thrombus in the right atrium (RA) extending into the right ventricle (RV) was made (LA, left atrium; LV, left ventricle). She was treated with peripheral intravenous tissue plasminogen activator (tPA) (total dose $100 \mathrm{mg}$ over 90 minutes). Repeat echocardiograms showed substantial resolution of the thrombus one hour after tPA and none was evident at 24 hours. The patient's symptoms resolved and a ventilation perfusion lung scan did not show any evidence of pulmonary emboli. We propose that this patient developed a calf vein thrombosis secondary to immobility, which then embolised in total at the time of her presyncopal episode to lodge in the right atrium. Mechanisms by which the thrombus could have been caught as it passed through the right heart include entrapment by the tricuspid valve apparatus or the Chiari network (congenital remnants of the right venous valve of the sinus venosus). In this patient Chiari nets, which can be seen by echocardiography, were not detected.

R A RUSK A KENNY J P BOURKE 